\title{
العمل الإنساني الدولي في حالات الطوارئ الصحية العامة (دور اللجنة الدولية للصليب الأحمر أنموذجاً)
}

\section{وهج خضير عباس}

أستاذ مساعد- كلية القانون- جامعة البصرة- العراق

wahag.khder@gmail.com 
www.refaad.com
المجلة الدولية للدراسات القانونية والفقهية المقارنة

International Journal of Legal and Comparative Jurisprudence Studies (LCJS)

Journal Homepage: https://www.refaad.com/views/LCJS/Home.aspx

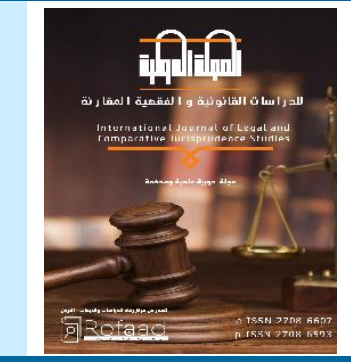

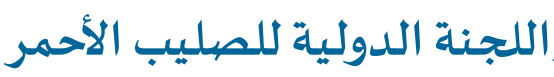

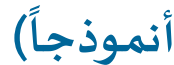

\section{وهج خضير عباس}

أستاذ مساعد- كلية القانون- جامعة البصرة- العراق

wahag.khder@gmail.com

DOI: https://doi.org/10.31559/LCJS2021.2.3.3 2021/9/6 2021/9/30: مراجعة البحثلام البحث البحث: 2021/20/20

\section{المللخص:}

العمل الإنساني يتضمن مجموعة من الإجراءات يقصد القائمون بها التخفيف من آلام ومعاناة المتضررين من جراء الأزمات الإنسانية

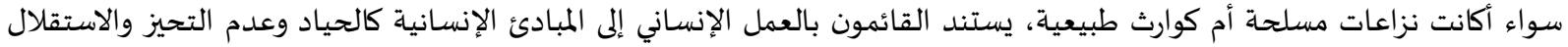

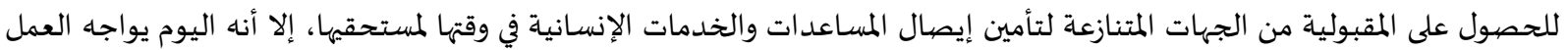
الإنساني الكثير من التحديات والعراقيل كأعمال الخطف والعنف ضد الأشخاص القائمين بالعمل الإنساني فضلاً عن انتشار الأوبئة والجوائح في بيئات غير آمنا مما يعرض حياة القائمين بالعمل الإنساني للخطر والكثير من الصعوبات التئي حالت التئ دون إيصال الاحتياجات الإنسانية للمتضررين في وقتها.

الكلمات المفتاحية: العمل الإنساني؛ حالات الطوارئ الصحية العامة؛ معايير الاستجابة الإنسانية؛ مدونة السلوك.

| ملمقدة:

تعاني الدول اليوم وفي كل أنحاء العالم حتى الدول المتقدمة منها من آثار الأوبئة والأمراض المعدية خاصة 19 COVID، الذي أثبت عجز المنظومة الصحية الدولية وضعفها مما أدى إلى إعلان حالة الطوارئ الصحية العامة ذات الاهتمام الدولي لضمان تحقيق أرفع مستويات التعاون الدولي لمجابهاة

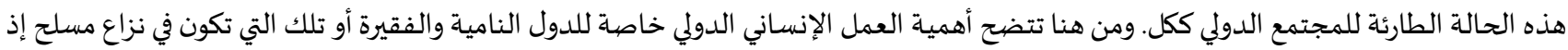
تزداد شدة ووطئة الكارثة الإنسانية المترتبة على حالة الطوارئ الصحية العامة بوجود النزاعات المسلحة وهنا يكون دور العمل الإنساني أكثر أهمية وفي

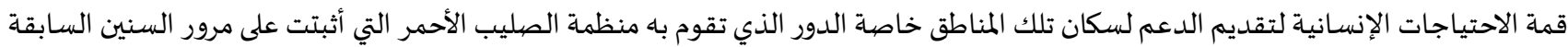
وإلى اليوم مدى أهمية دورها في تخفيف المعاناة والآلام لسكان المناطق التي تعاني من النزاعات المسلحة خاصية في فترة الطوارئ الصحية العامة.

$$
\text { أهمية الدراسـة: }
$$

تكمن أهمية الدراسة في بيان مدى أهمية العمل الإنساني الدولي في حالات الطوارئ الصحية العامة ذات الاهتمام الدولي وتأثيره في الاستجابة

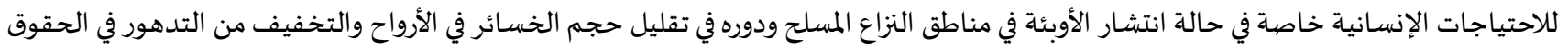
الإنسانية الصحية، فضلاً عن بيان أهم المؤسسات الإنسانية والوثائق الدولية الداعمة للعمل الإنساني الدولي في النزاعات المسلحة خاصة خاصية الميثاق الإنساني (اسفير) للمساعدات الإنسانية لمجابهة ومواجهة هذه الحالات الطارئة. 
أهداف الدراسـة: (1)

تهدف الدراسة بيان الأمور الآتية:

تحديد مقومات العمل الإنساني الدولي ومبادئه وأساسياته التي يقوم عليها لبيان أهميتها وكيفية ممارستها في مواجهة حالات الطوارئ الصحية

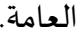

العمل على إيجاد وتحديد أفضل الوسائل والآليات لدعم ممارسة العمل الإنساني والتطبيق الصحيح لمعاييره وأسسـه وفسح المجال أمام مؤسساته ضمن منظور دولي يضمن تذليل معوقات مباشرة العمل الإنساني الدولي وبالتالي الحفاظ والعمل على تلبية الاحتياجات الإنسانية في حالة الطوارئ الصحية العامة.

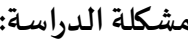

تكمن المشكلة في أنه على الرغم من أهمية العمل الإنساني الدولي في مناطق النزاعات المسلحة خاصة في حالات الطوارئ الصحية العامة، فضلاً

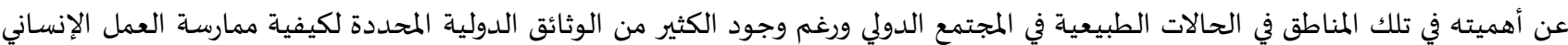
ووجود الكثير من المؤسسات الدولية القائمة بهذا العمل إلا أن الوضع الحالي في مناطق النزاع خاصة في ظل انتشار الجوائح كفيروس كورونا ينذر

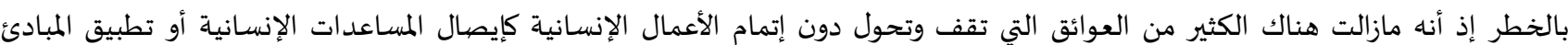

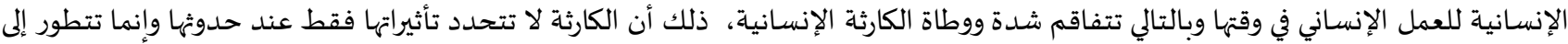

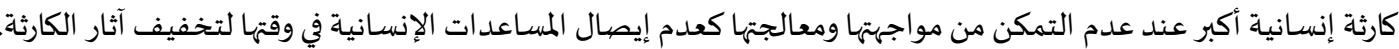

أسئلة الدراسـة:

سيتم خلال هذه الدراسـة الإجابة عن الأسئلة الآتية: من هي الجهات الفاعلة التي تضطلع للقيام بالعمل الإنساني في مناطق النزاع المسلح عند انتشار الأوبئة؟ ماهي حالات الطوارئ الصحية المعقدة؟ ماهي أهم المعوقات التي تواجه الجهات الفاعلة عند ممارسة العمل الإنساني في حالات الطوارئ الصحية العامة؟

منهجية الدراسـة: استخدم الباحث المنهج الوصفي ومنهج التحليل القانوني في إيراد النصوص القانونية والاتفاقيات الدولية فضلاً عن استخدام المنهج التاريخي للبحث في التطبيقات العملية للمواضيع ذات الصلة بموضيوع الدراسة. هيكلية الدراسـة: للاحاطة بموضوع الدراسة سوف تقسم إلى التقسيمات الآتية: المبحث الأول: مفهوم العمل الإنساني الدولي. المطلب الأول: التعريف بالعمل الإنساني الدولي. المطلب الثاني: المعايير الدنيا للاستجابة الإنسانية على وفق الميثاق الإنساني (اسفير) المطلب الثالث: مبادئ ممارسة العمل الإنساني

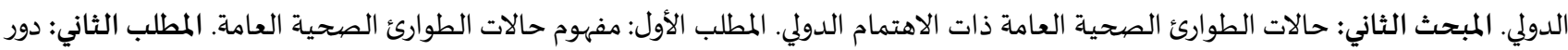
الجهات الفاعلة في مجال العمل الإنساني عند إعلان حالات الطوارئ الصحية العامة(منظمة الصليب الأحمر نموذجاً). المطلب الثالث: مدونة قواعد

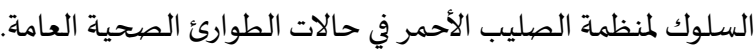

المبحث الأول: مفهوم العمل الإنساني الدولي

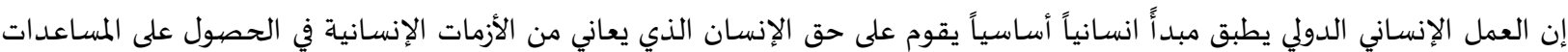

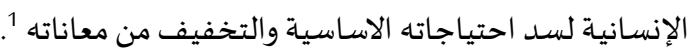
وللإحاطة بمفهوم العمل الإنساني الدولي سنتناوله على وفق التفصيل الآتي:

$$
\text { المطلب الأول: التعريف بالعمل الإنسـاني الدولي }
$$
العمل الإنساني الدولي هو: التدابير التي يتم اتخاذها بهدف إنقاذ الأرواح والتخفيف من المعاناة والآلام والحفاظ على الكرامة الإنسانية اثناء وبعد

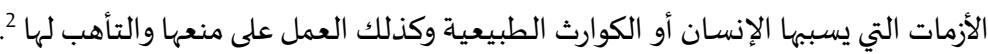

"المعيار الانساني الاساسي ,ملحق اسئلة ارشادية لرصد الاعمال الاساسية والمسؤوليات التنظيمية، ص 362، متاح على الموقع الالكتروني تمت الزيارة الساعة 3:00 مساءا في 2021/6/2: https://kayaconnect.org/course/info.php?id=490 2 المعايير الانسانية الاساسية المتعلقة بالجودة والمسائلة، تحالف المعايير الانسانية الاساسية ومجموعة (Groupe URD) ومشروع اسفير ، 2014 ، ص 36. 
تتألف منظومة العمل الإنساني من كيانات تنظمية مهمتها القيام بالعمل الإنساني كالمنظمات غير الحكومية المحلية، الوطنية والدولية، الوكالات

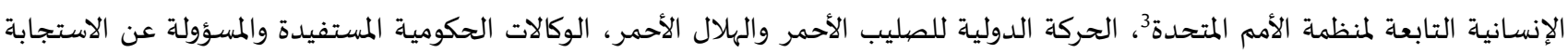
للأزمات، المؤسسات الإنسانية التابعة للمنظمات الحكومية الدولية الاقليمية وأخيراً الوكالات والمكاتب الحكومية الممولة للعمل الإنساني. تقوم منظومة العمل الإنساني بالأعمال الآتية:

تقديم الاغاثة العاجلة عند الكوارث الفجائية الحدوث ${ }^{4}$ التي تعجز قدرة الدولة والجهات الفاعلة المحلية عن الاستجابة لها.

تلبية الاحتياجات الإنسانية الرئيسية للسكان المتضررين في حالة النزاعات المسلحة أو الكوارث الطبيعية المتكررة. 5 العمل على تطوير مقدرة الشعوب المتضررة وضمان مشاركتهم لتحقيق التنمية المستدامة والعمل على سد احتياجاتهم الإنسانية ذاتياً في المستقبل تقع مسؤولية تمين العمل الإنساني وحمايته في الدرجة الأولى على الدولة المتضررة الموجود فيها الكادر الممارس للعمل الإنساني7 مع مراعاة

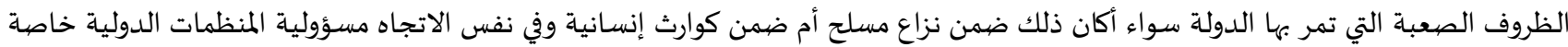
الأمم المتحدة نفسها وهنا تبرز أهمية تبادل المعلومات بين الجهات الحكومية المختصة في الدولة والوكالات الإنسانية للتمكن من التحديد الصريح للمخاطر و وضيع الخطط الأمنية لتجاوزها 8. كما شكلت التكنولوجيا الحديثة باستخدام الإنترنت أيضاً دوراً في ممارسة العمل الإنساني عن بعد خاصها فئا في البيئات غير الأمنة حفاظاً على حياة

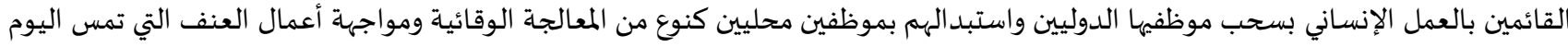

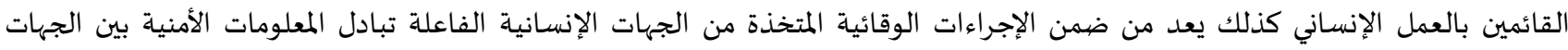

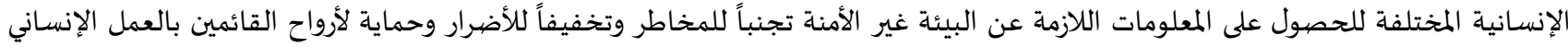
وسلامة المؤن والمساعدات الإنسانية 9. إلا أنه رغم أهمية العمل الإنساني الدولي لمساعدة وتخفيف الآلام لضحايا النزاعات المسلحة واضطالاع الجهات المهات الإنسانية الفاعلة بذلك توجد الكثير من الصعوبات والعقبات التي تحول دون إتمام العمل الإنساني وتعرض القائمين بها إلى الخطر والتي يمكن إجمالها بما يلي:

هيئات الامم المتحدة المختصة بالعمل الانساني هي:

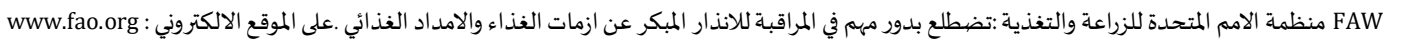

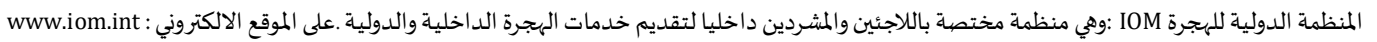

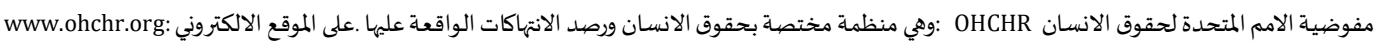

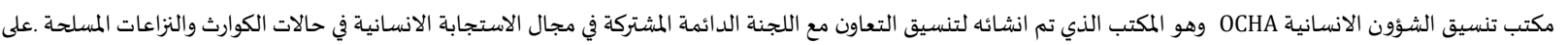

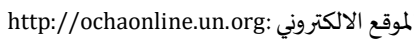

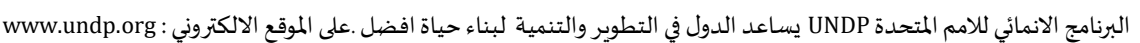

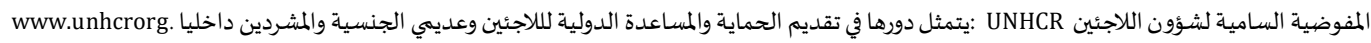

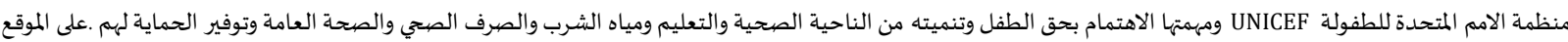

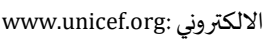

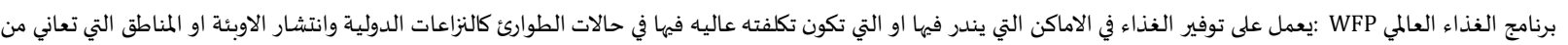

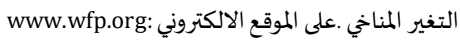

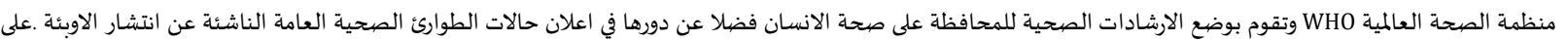
www.who.org: الموقع الالكتروني الصجة العالمة

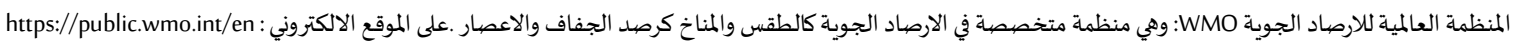

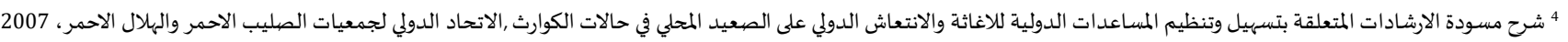

5 وضع منظومة العمل الانساني , شبكة التعلم الايجابي للمساءلة والاداء، 2015، ص 19، متاح الساعة 10:00 صباحا في 2021/5/31 على الموقع الالكتروني: https://www.alnap.org/system/files/content/resource/files/main/sohs2015-arabic.pdf 6 اللجنة الدائمة المشتركة بين الوكالات ومكتب تنسيق الشؤون الانسانية التابع للامم المتحدة 2007، وقت الزيارة 9:00 ص في 6 /2021/8، متاح على الموقع الالكتروني http:ochaonline.un.orglcmcs/guideline: 7 ان مسالة تاكيد الدول على سيادتها مازالت الى الان تؤثر سلبا في تاخير او رفض الاستجابة الدولية للاحتياجات الانسانية وخاصة ازداد ذلك في الوقت الحالي تحت حجة منع الارهاب و اعمال حفظ المالي

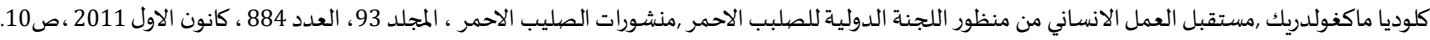

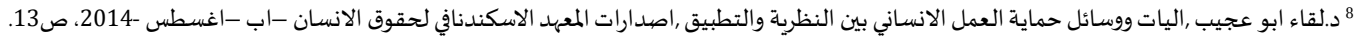

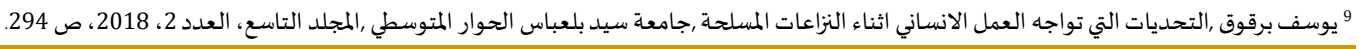


أسباب تدهور العمل الإنساني:

طبيعة النزاعات المسلحة الحديثة: أغلب النزاعات المسلحة في الوقت الحاضر هي نزاعات مسلحة غير دولية تقوم غالباً في المدن فكان لازدياد عدد

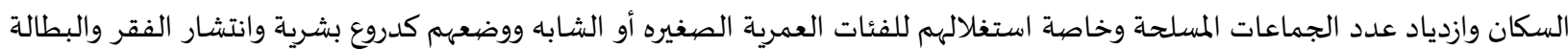
وتفشي الفساد في مؤسسات الدولة وعدم التمكن من السيطرة على العنف المسلح للجماعات المسلحة كلها كانت تمثل مزيداً من التحديات في

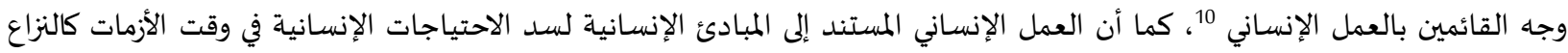

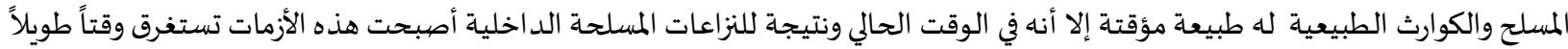
مما يترتب عليه تصدع كبير في البنى التحتية للدول التي تعاني منها فيتفكك القانون وتضعف مؤسسات الدولة عن تقديم خدماتهاتها بتلبية الاحتياجات الاساسية للسكان كالغذاء والماء والدواء فتتدهور الحالة الصحية فضلاً عن انتشار الأوبئة في مثل هذه البيئات يجعل الكل ضمن فئن معارك كبيرة فمن جهاة تردي الوضع وتدهور البنى التحتية بسبب النزاع المسلح ومن جهة أخرى تفشي الجوائح التي حتى في الدول التي تعيش في

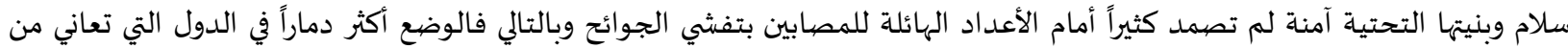

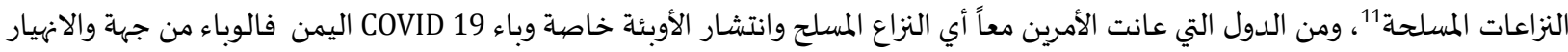
الاقتصادي وانهيار المؤسسات وتدمر البنى التحتية فضلاً عن ذلك أن 80\% من سكانه هم من النساء والأطفال مما شكل أزمة إنسانية خطيرة

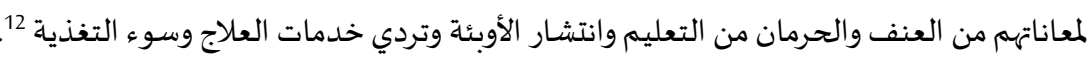

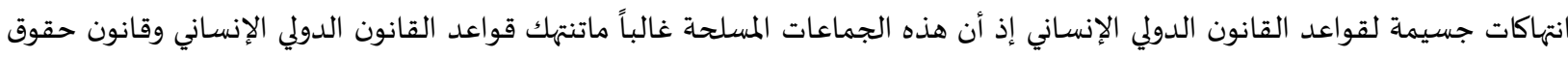

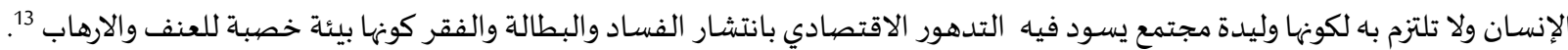
من الصعوبات والتحديات في وجه العمل الإنساني هو عدم إيصال أو تأخر إيصال المساعدات الإنسانية لسد الاحتياجات الملحة في الوقت

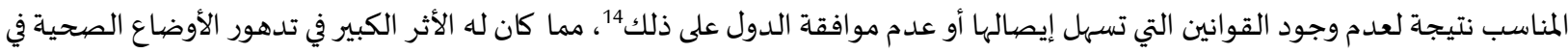

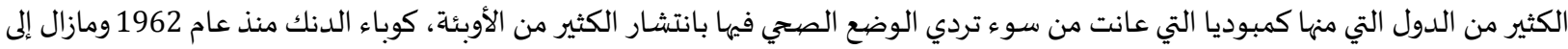

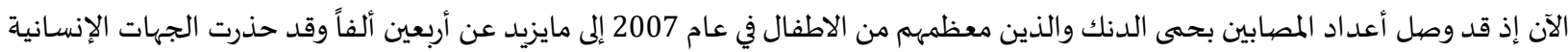
الفاعلة وفي مقدمتها اللجنة الدولية للصليب الأحمر من تدهور الأحوال خاصة بظهور انفلونزا الطيور وغيرها لذا لابد من الاستجابة الإنسانية

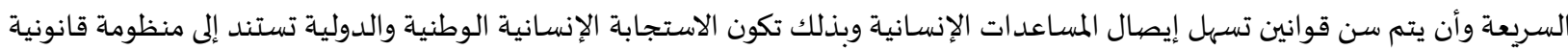

سليمة لسد الاحتياجات الإنسانية في وقت مناسب حتى لانئ لا تتفاقم الأزمة 15. عسكرة العمل الإنساني: إن العمل الإنساني يقدم من قبل الجهات الإنسانية إلا أنه في الوقت الحاضر توجد العديد من التحديات والصعوبات

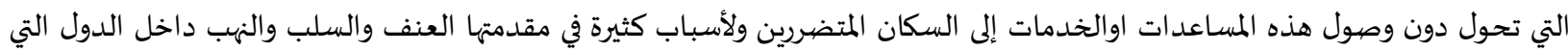
تعاني من النزاعات المسلحة غير الدولية إذ يتعرض العاملون في مجال العمل الإنساني للاختطاف أو الابعاد القسري أو أعمات أعمال العنف من قبل فيل

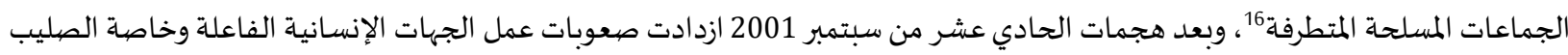

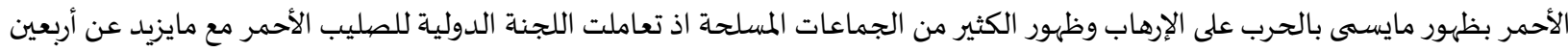

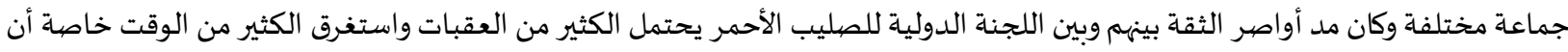

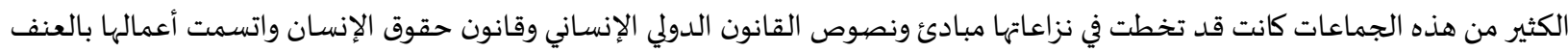

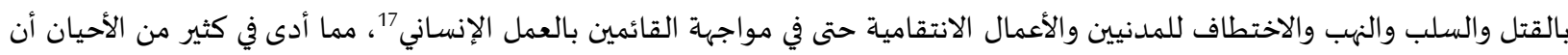

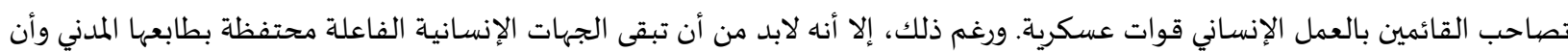

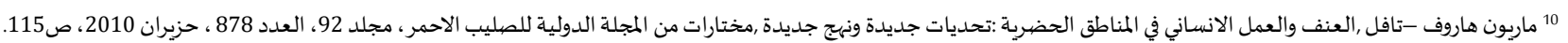

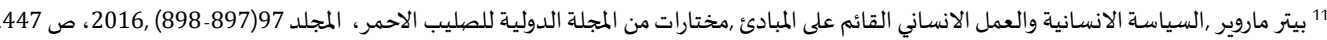

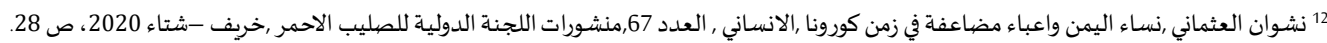

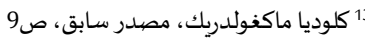

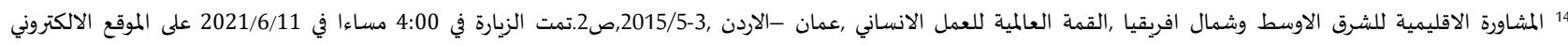
https://www.alnap.org/system/files/content/resource/files/main/scoping-paper-mena-regional-consultation-\%5Barabic\%5D.pdf

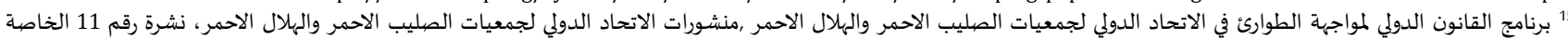

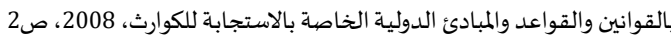

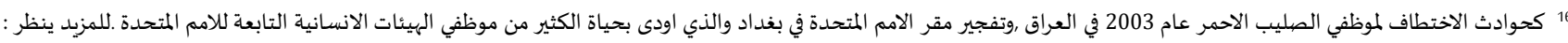

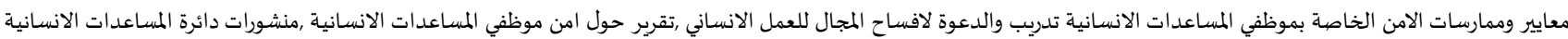
التابعة للمفوضية الاوربية (ECHO)،

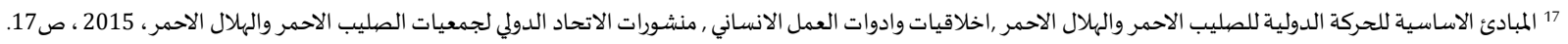


يكون التقديم للمساعدات الإنسانية من قبل متطوعي وموظفي الوكالات الإنسانية وليس من قبل القوات العسكرية، وأن يكون وجود هذه

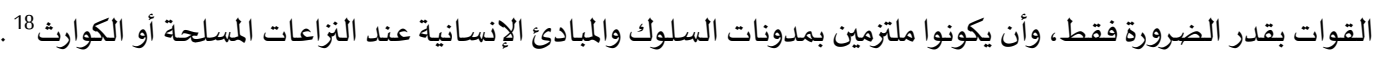
ولقد انشات لتامين الحماية للقائمين بالعمل الإنساني الكثير من الشركات الأمنية الخاصة إلإ أنها ورغم أهمية توفير الحماية للعاملين في مجال

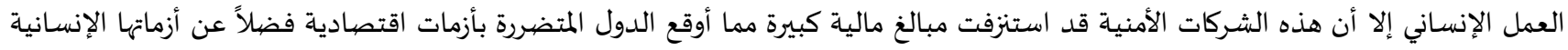

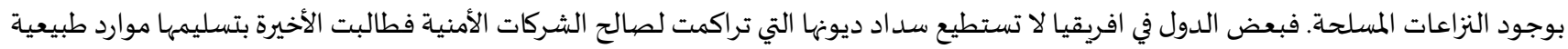

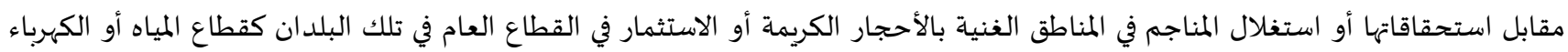
وبالتالي كان لعسكرة العمل الإنساني آثاراً سلبية كبيرة 19.

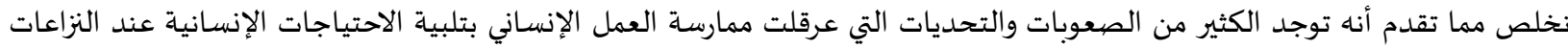

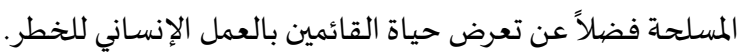

المطلب الثاني: المعايير الدنيا للاستجابة الإنسانية على وفق الميثاق الإنساني (اسفير) إن الميثاق الإنساني اسفير يتضمن الإشـارة إلى معتقدات مشتركة ومبادئ شائعة في حالات الكوارث والأزمات الإنسانية. تتميز أهم المعايير الدنيا المانيا

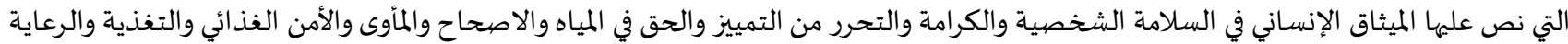

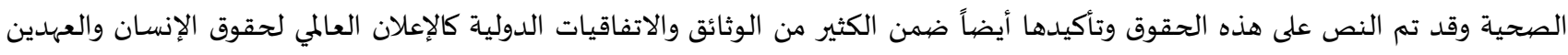
الدوليين والاتفاقية الدولية للقضاء على التمييز العنصري بكافة أشكاله ...الخ كذلك تم الاشارة لهذه الحقوق ضيمن مبادئ وتوجيهات الأمم المتحدة

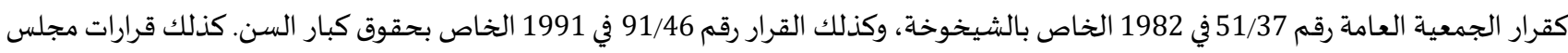
الأمن بشأن العنف الجنسي الذي تتعرض له النساء في النزاعات المسلحة كالقرار رقم 1325 في عام 2000 بشأن النساء والسلام والأمن.

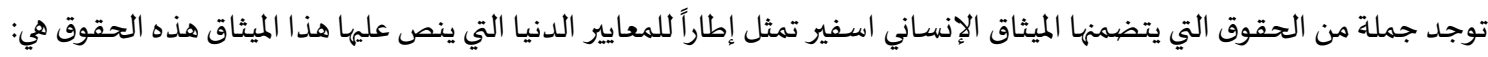

$$
\text { الحق الحق في الحياة بكرامة. }
$$

كذلك تم الاشارة إلى المعاييرالدنيا في الميثاق الإنساني اسفير ضيمن نصيوص القانون الدولي الإنساني وضمن هذا السياق تعد اللجنة الدولية

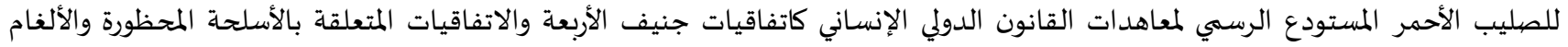

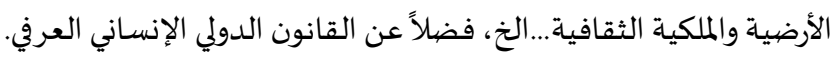

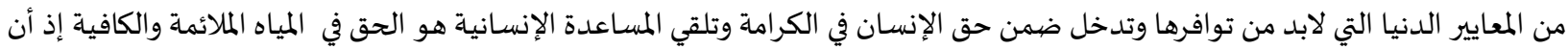

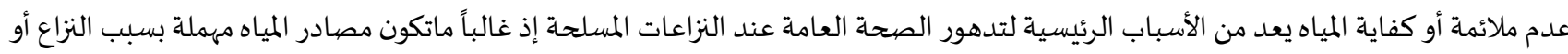

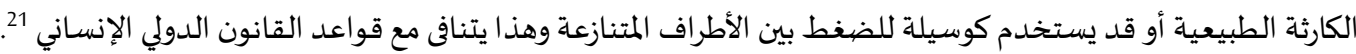

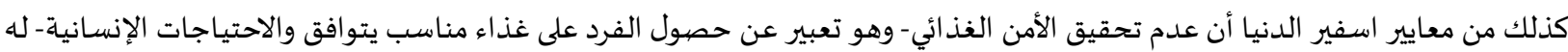

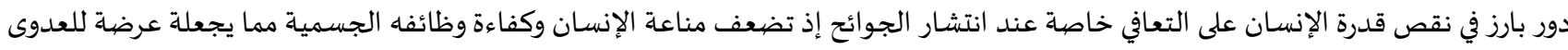
وتزيد من قابليته على الإصابة بالأمراض المزمنة وتؤثر على سـائر حقوقة الإنسانية الأخرى كحقه في العمل وغيره من الحقوق الإنسانية الأخرى وبالتالي

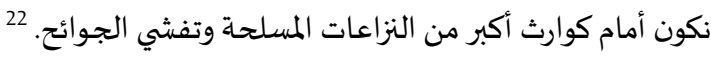

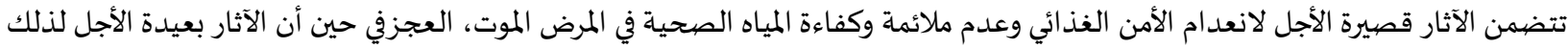

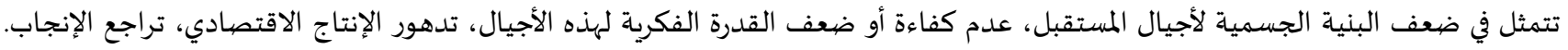

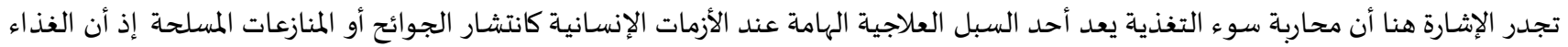

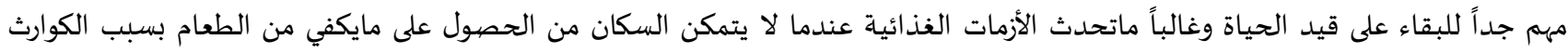

18 اللجنة الدائمة المشتركة بين الوكالات ومكتب تنسيق الشؤون الانسانية التابع للامم المتحدة 2007، وقت الزيارة 9:00 ص في 6 /2021/8، ص 13، متاح على الموقع الالكتروني http:ochaonline.un.orglcmcs/guideline: 19 د.لقاء ابو عجيب، مصدر سابق ص43.

20 المعيار الانساني الاساسي، ملحق اسئلة ارشادية لرصد الاعمال الاساسية والمسؤوليات التنظيمية مصدر سابق، صابل ص 352.

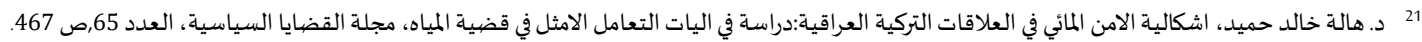

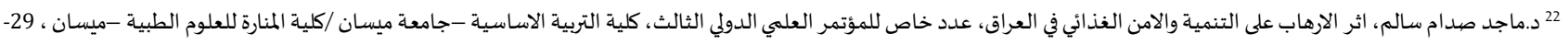

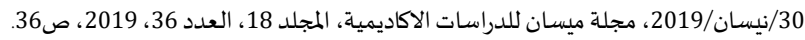


الطبيعية أو النزاعات المسلحة وعدم الاستقرار السياسي أو عند انتشار الأوبئة والجوائح. لذا لابد من توفير الأمن الغذائي أي الحصول على غذاء صيحي وكاف في الحاضر والمستقبل 23.

في الوقت الراهن فإن ضمان تأمين المساعدات الإنسان بتوفير الحاجات الإنسانية القصيرة الأجل من إمدادات المياه والغذاء الكافي لمن هم ضمن

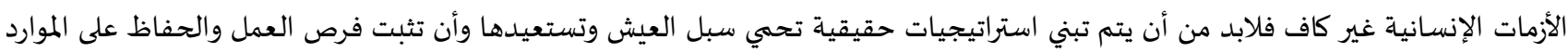

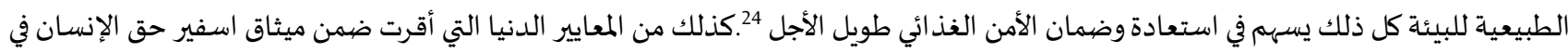

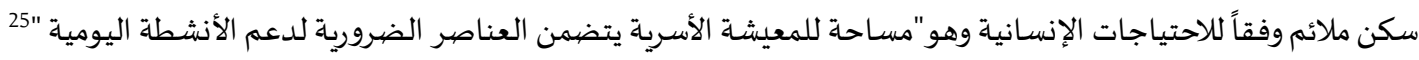

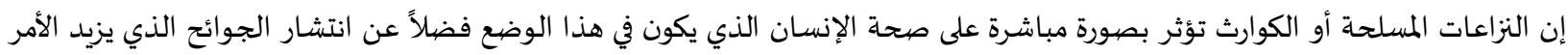

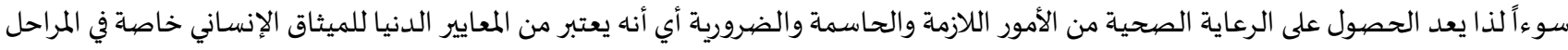

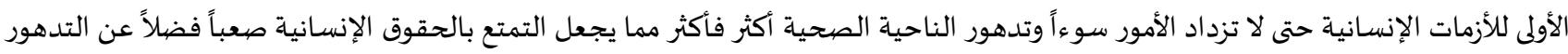

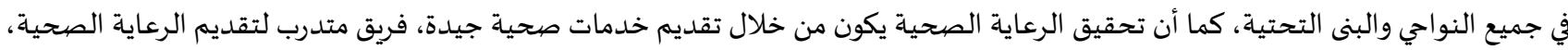

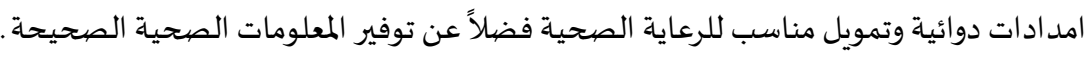

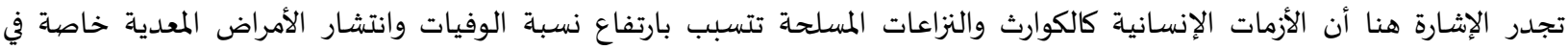

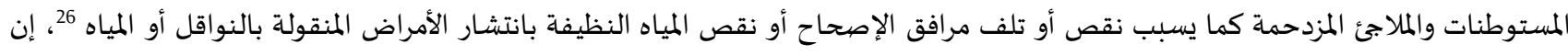

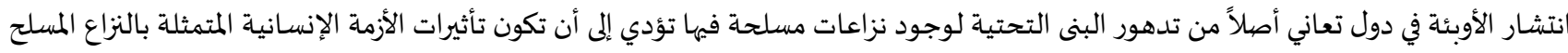

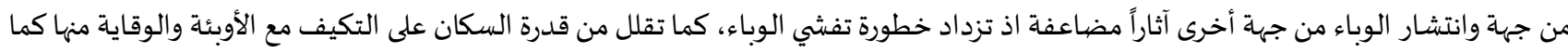

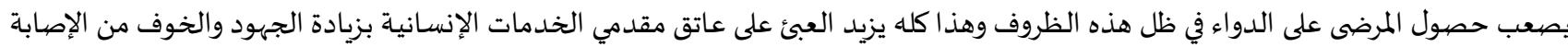

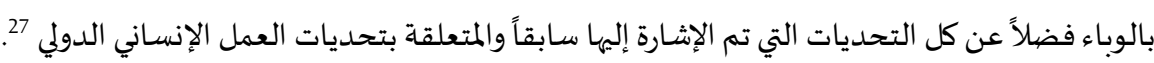

إذن المعايير الدنيا التي أقرها الميثاق الإنساني (اسفير) تمثل الاحتياجات الإنسانية الضرورية للبقاء على قيد الحياة وصهون الكرامة الإنسانية .

المطلب الثالث: مبادئ ممارسـة العمل الإنساني الدولي

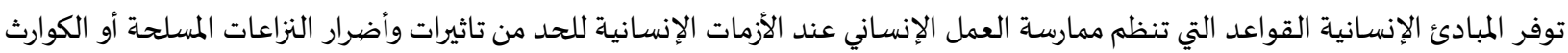

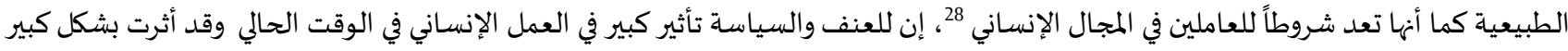

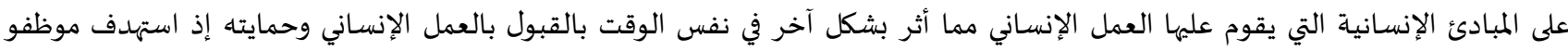

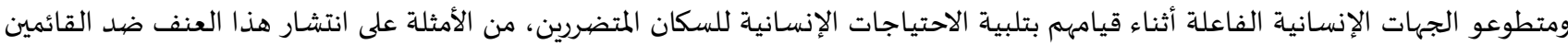

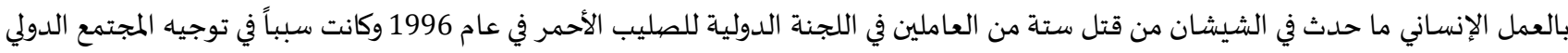
وتنبيها إلى الخطر الكبير الذي يتعرض لله موظفو الجهات الإنسانية الفاعلة 29.

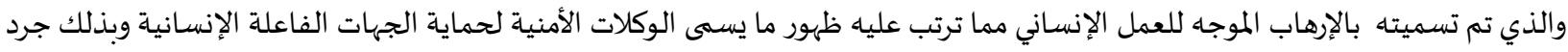

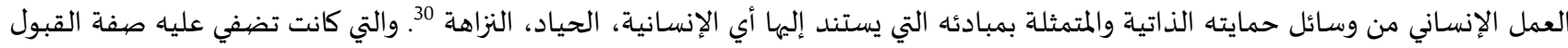

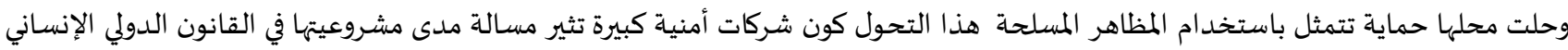

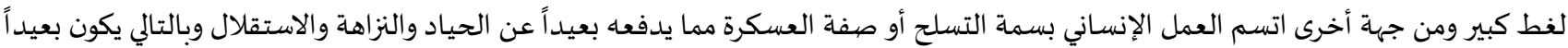
عن القبول .

إذ يقصيد بالمبادئ الإنسانية وفقاً لقرار الجمعية العامة للامم المتحدة 182/46 أن العمل الإنساني يجب أن يقدم مستنداً إلى المبادئ الإنسانية

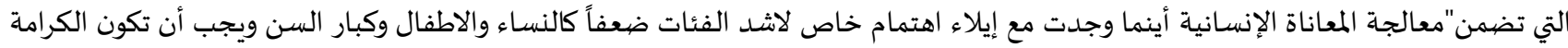
والحقوق محل احترام وحماية" إن هذه المبادئ الإنسانية هي:

23 اداة عملية للعاملين في المجال الانساني , ALL IN DIARY ، الاصدار الثاني، يناير ، ص 22.تمت الزيارة الساعة 4:00 صباحا في 2021/6/4 على الموقع الالكتروني : https://www.eldis.org/document/A43456

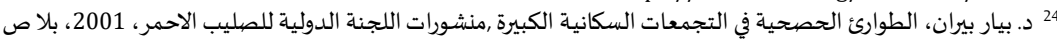

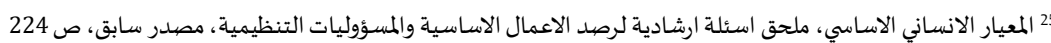

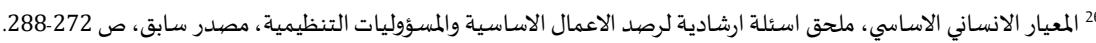

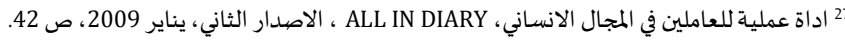

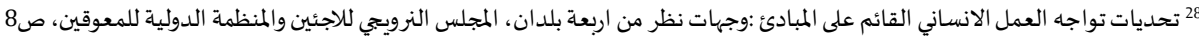

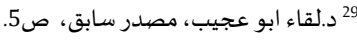

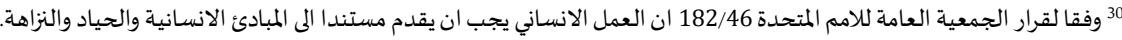

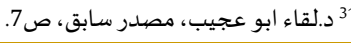


عدم التحيز: ويعني تقديم المساعدات الإنسانية للمتضررين حسب الاحتياجات الإنسانية الأكثر إلحاحاً دون مراعاة أي جنسية أو وضع قانوني

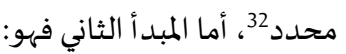

الحياد: ويقصيد باه ممارسة العمل الإنساني دون المشاركة في الأعمال العسكرية أو الانحياز الى أي طرف نتيجة لبعدد سياسي أو ديني أو عرقي.

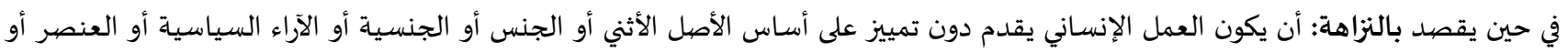
الدين. كما أكد القرار أعلاه احترام سيادة الدولة وسلامتها الأقليمية ووحدتها الوطنية33 احتراماً كاملاً لذا يعد الحصول على موافقة الدولة المتضررة أحد شروط أداء العمل الإنساني. تم إنشـاء مايصطلح على تسميته مثلث الأمن للعمليات الإنسانية الذي يعتمد على المبادئ الإنسانية (الحياد، عدم التحيز، الاستقلال) للحصول

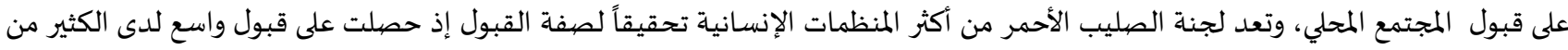

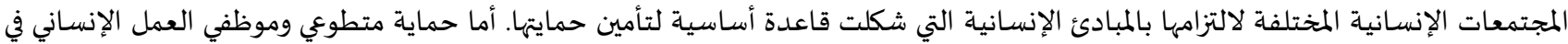

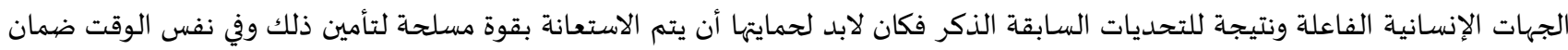

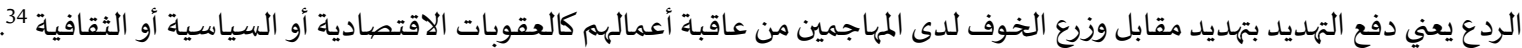
وهي مجموعة من الإجراءات والخطوات الأمنية التي تعزز الاستراتيجيات الأمنية للوكالات الإنسانية. للجنة الدولية للصليب الأحمر أهمية كبيرة ومنذ نشاتها باستنادها إلى مبادئ العمل الإنساني الواضحة التهوية التي تقوم على عدم التحيز والمحايده

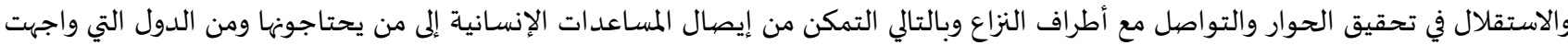

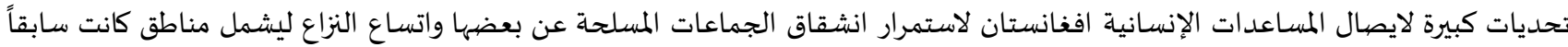

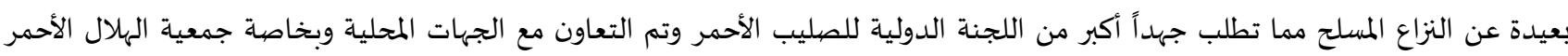

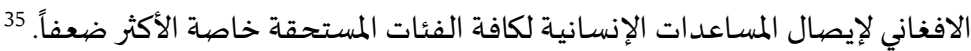
إذن المبادئ الإنسانية التي كان يستند إلها العمل الإنساني في الحصيول على القبول من قبل الحكومات والمجاداميع المسلحة وبالتالي ضمانمان عدم

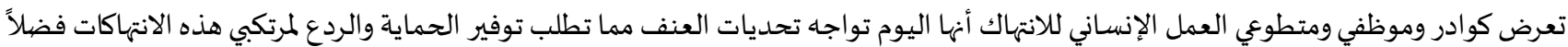
عن استنادها للقبول القائم على المبادئ مما قد يخلط العمل الإنساني بالتوجهات السياسية للحكومة الوطنية تحت ذريعة حماية القائمين بالعمل الإنساني أي التاثير في عقول وقلوب مواطنيها أي مايسمى (نظرية كسب القلوب والعقول) على أسـاس أهنا تضمن وصهول المساعدات الإنسانية للمتضررين مما يؤدي إلى الاختلاط بين الإتجاه السياسي والعمل الإنساني وبالتالي تعاظم وتكاثف العنف ضد الطواقم الطبية والقائمين على العمل

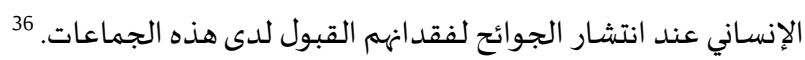

\section{المبحث الثاني: حالات الطوارئ الصحية العامة ذات الاهتمام الدولي}

سنتناول ضمن هذا المبحث مفهوم حالات الطوارئ الصحية العامة وبيان اهم الجهات الفاعلة لمواجهتها.

المطلب الأول: مفهوم حالات الطوارئ الصحية العامة حق الإنسان في الصحة من الحقوق الاسـاسية إذ توجد العديد من الوثائق والاتفاقيات الدولية المؤكدة ليس لكونه أحد الحقوق الإنسانية

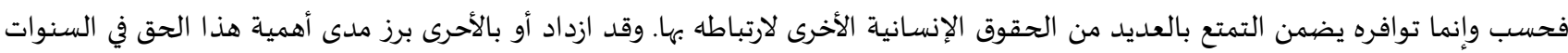

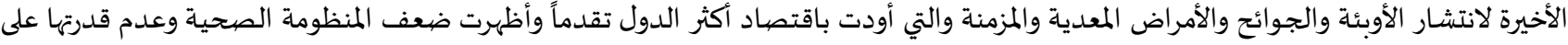

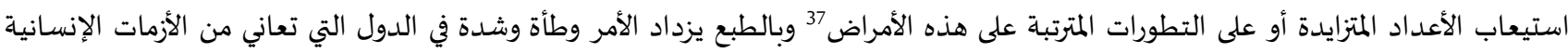
خاصة النزاعات المسلحة لأن أصلاً حق الصحة فيها يتعرض للانتهاك بسبب ظروف الحسب الحرب فماذا لو اجتمعت الحروب مع انتشار الجوائح فالدول التي

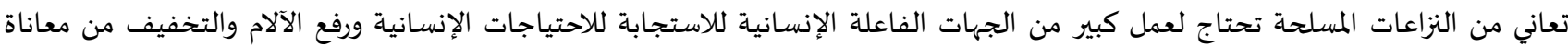

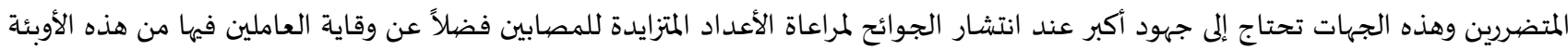

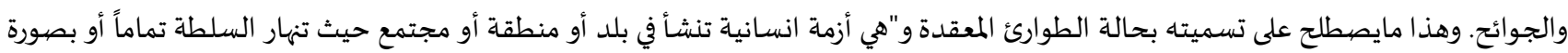

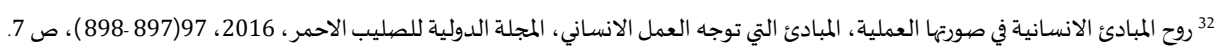

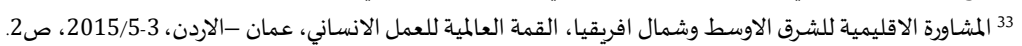

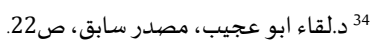

(35 35

33 روح المبادئ الانسانية في صورتها العملية، المبادئ التي توجه العمل الانساني، المجلة الدولية للصليب الاحمر، 2016، 927(897 -898 ) مصدر سابق، ص 10. ${ }^{37}$ Lucia Mullen Christina Potter,Llawrence O Gostin,Anita Cicero,Jennifer B Nuzzo ,An analysis of International Health Regulations Emergency committees and Publice Health Emergency of International Concern Designation,2020 ;5:e002502.doi:10.1136/bmjgh-2020-002502,p2. 
ضخما نتيجة لصراع داخلي أو خارجي وتتطلب استجابة دولية تتجاوز ولاية أو قدرة أية وكالة قائمة بذاتها " 38 إذ يجتمع هنا النزاعات المسلحة وانتشار الأوبئة وكلاهما يستنزف حقوق الإنسان.

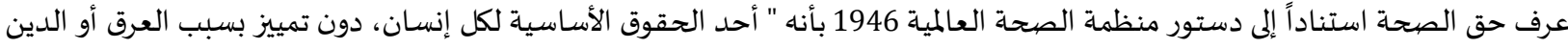

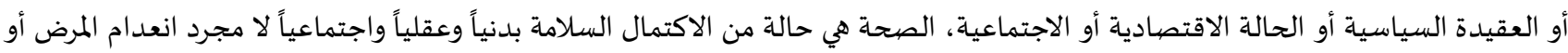

إن للقانون الدولي العام دور كبير في المراقبة العالمية للأمراض المعدية فترتب على ذلك تنسيق أنظمة الحجر الصبحي وتسهيل تبادل المعلومات

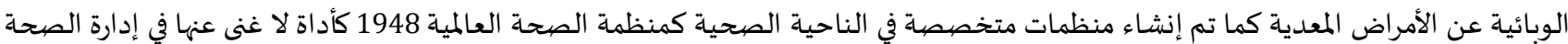

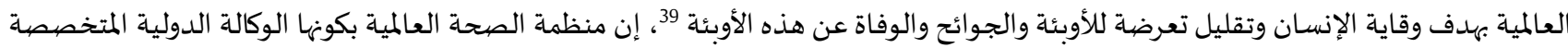

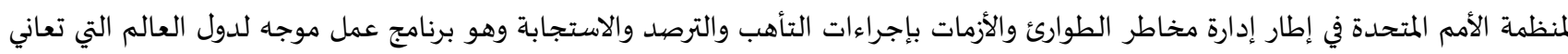

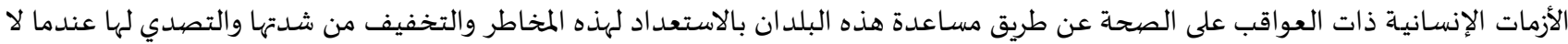
تتمكن الدول المتضررة من تحمل أعبائها. 40 تقاس حالات الطوارئ الصحية العامة بما تخلفه من وفيات وأمراض واعاقات وأن لمنظمة الصحة العالمياة دور رئيسي في إدارة المخاطر الصحية

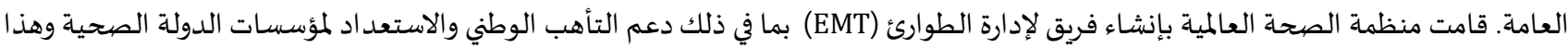

هو من يقوم بتحديد شدة الحالة الطارئة الصحية. 41

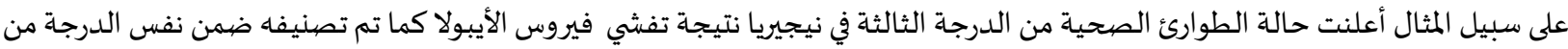

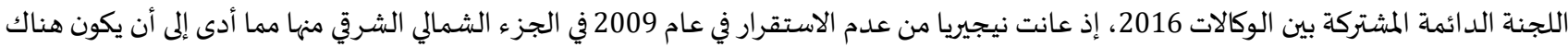
نزوح يصل إلى مايزيد عن 2.2 مليون شخص وقامت منظماة الصحة العالمية -الوكالة المتخصصية التابعة للأمم المتحدة والتي تعتمد اللوائح الصحية بتحديد طرق مكافحة وتسمية الأمراض وأسباب الوفاة وتحديد معايير التشخيص والمعايير ذات الصيلة بالئل بالسلامة ونقاء وفاعلية المنتجات الحياتية والإعلان عنها كما تقدم التوصيات في الشؤون الصحية،، 42 كما اعلنت منظمة الصحة العالمية في 30/كانون الثاني /2020 حالة الطوارئ الصحية العامة عن تفشي فيروس 19 COVID والتي تثير قلقاً دولياً وخطراً كبيراً على كل الدول خاصة الداعة الدول ذات الأنظمة الصحية الضعيفة. 43

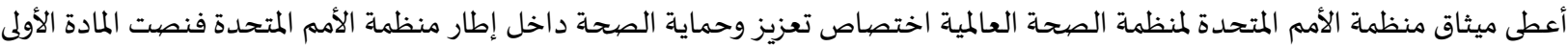

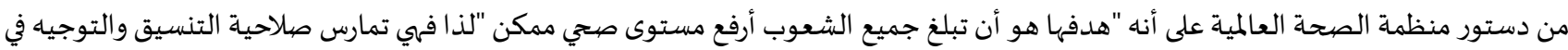
مجال العمل الدولي الصحي فلجمعية منظمة الصحة العالمية إصدار قرارات تشريعية لمكافحة الأمراض المعدية دولياً. في عام 2009 عقدت منظمة الصحة العالمية اجتماعا مع لجنة الطوارئ وبعد عدة مشاورات أعلن المدير العام بموجب اللوئح الصحية 2005 الصدية بوجود حالة صحية طارئة عامة انفلونزا (H1N1) تعد خطراً على الصحة العامة للدول لانتشارها دولياً وتم رفع مستوى الوباء للمرحلة الخامسة

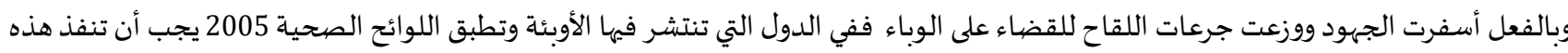

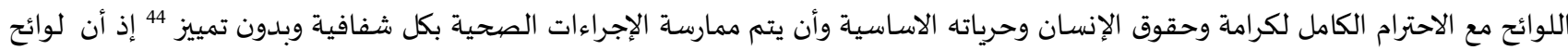
منظمة الصححة العالمية لمكافحة انتشار الأوبئة 45 تفرض الاحتران وحترباته المخاط المانبين بها وبالتالي بطلان تصرف أي دولة تخالف ماورد بها وتثبت بذلك مسؤولية

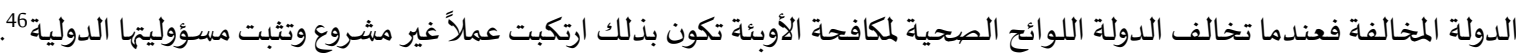

https://www.thenewhumanitarian.org/ar/report/1657: 38 تعريف وضعته اللجنة الدائمة المشتركة بين الوكالات 1992، الساعة 3:002 مساءا، في 2021/6/6 على الموقع الالكتروني ${ }^{39}$ Obijiofor Aginam, International law and communicable diseases ,Global Public Health and International Law ,Bulletin of the World Health Organization 2002, $80(12), \mathrm{p} 946$

40 ادارة مخاطر الطوارئ والاستجابة الانسانية تقرير 2014:Po13-2014 ، منشولرات منظمة الصحة العالمية رجنيف-سويسرا، على الموقع الالكتروني ${ }^{41}$ Muhammad A.S0ghaier ,Khawaja MI Saeed,and Khushhal K.Zaman ,declared a Public Health Emergency of International Concern (PHEIC)twice in 2014;Polio and Ebola at top ,AIMS Public Health .2015;2(2):218-222.

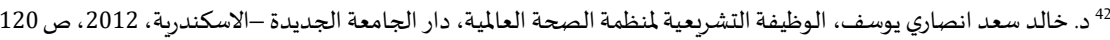

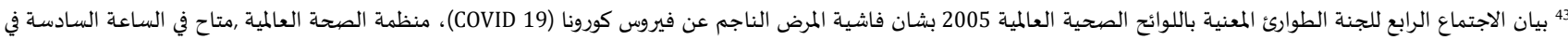
2021/10/8 20لى الموقع الالكتروني: -health-regulations-(2005)-emergency-committee-

https://www.who.int/ar/news/item/11-12-1441-statement-on-the-fourth-meeting-of-the-international (regarding-the-outbreak-of-coronavirus-disease-(covid-19

${ }^{44}$ Advancing the right to health: the vital role of law ,Public health emergencies SUMMARY POINTS,p 169

https://www.researchgate.net/publication/324531402_Advancing_the_Right_to_Health-The_Vital_Role_of_Law على الموقع الالكتروني ${ }^{45}$ Legal Responses to Health Emergencies, The Law Library of congress ,Global Legal Research Center (202)707-6462(phone).(866)550-0442(fax).law@loc.gov http://www.law.gov p1.

46 
إذن على وفق ماتقدم تتكاتف الجهود الدولية للجهات الإنسانية الفاعلة وتبذل مزيداً من الجهد ضمن إطار ما يسمى بالحالات الطارئة المعقدة إذ إذ إذا

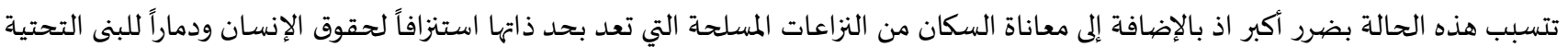
وفي مقدمتها مؤسسات الدولة المقدمة للرعاية الصحية يسبب انتشار الأوبئة آثاراً سلبية مضاعفة المباءة.

المطلب الثاني: دور الجهات الفاعلة في مجال العمل الإنساني عند إعلان حالات الطوارئ الصحية العامة (منظمة الصليب الأحمر أنموذجاً)

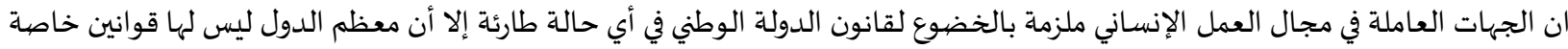

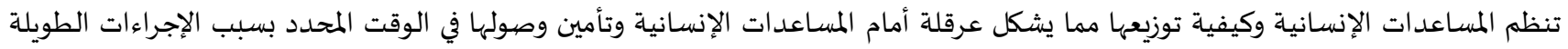
والمعقدة.

من المبادئ السائدة في القانون الدولي العام أنه على الجهات المتنازعة سواء أكانت الدول في حالات النزاعات الدولية المسلحة أو الدولة الوطنية

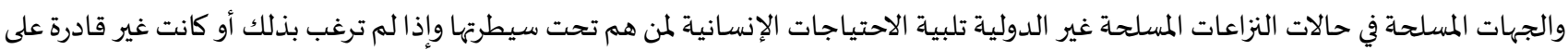

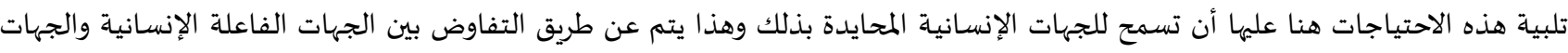
المتنازعة.

تقوم الجهات الإنسانية الدولية الفاعلة بدور كبير في العمل الإنساني وتحقيقه لأهدافه وتعد منظمة الأمم المتحدة المنظمة الحكومية الدولية

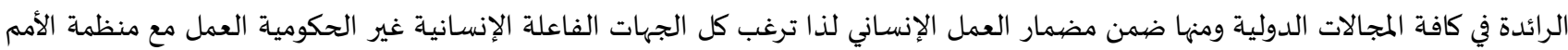
المتحدة لمواجهة الأزمات الإنسانية والعمل في جو يضمن سيادة واستقلال واحترام الشركاء وعلى منظمة الأمم المتحدة احترام استقلال هذه الجهات

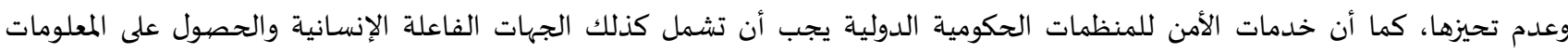
الصحيحة لمواجهة هذه الأزمات. 48

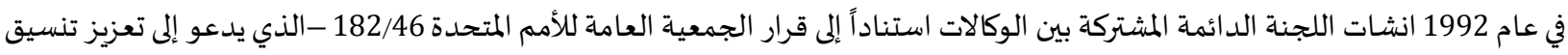
المساعدات الإنسانية- وقد تم تاكيد دور هذه اللجنة استناداً إلى القرار 57/48 بصفتها الآلية الرئيسية للتنسيق بين الوكالات في مجال المساعداعدة

إن النزاعات المسلحة سواء أكانت نزاعات مسلحة دولية أو غير دولية وما تتضمنها من انتهاكات جسيمة للقانون الدولي الإنساني والقانون الدولي لحقوق الإنسان أوجدت مجموعات كبيرة من الأشخاص ضمن وضع مؤلم من النقص في أبسط الاحتياجات الإنسانية كالغذاء والدواء والمأوى

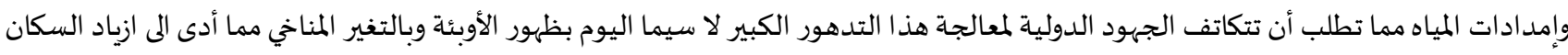
المتضررين، وفي عام 1996 تم الاتفاق بين الأمم المتحدة واللجنة الدولية للصليب الأحمر على وضع نقاط توجيهية لموظفي الأمم المتحدة لتطبيق واحترام قواعد القانون الدولي الإنساني وتامين سلامتهم، إذ يعاني العاملون في المجال الإنساني عند تلبيتهم للاحتياجات الإنسانية في البيئات غير الأنيات الأمنة إلى إلى الاعتقال والتعذيب والخطف والحرمان من حرية التنقل وضعف الاعتراف القانوني بمؤسستهم فضلاً عن التعرض لإمدادات المساعدات الإنسانية

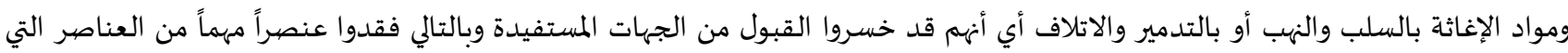

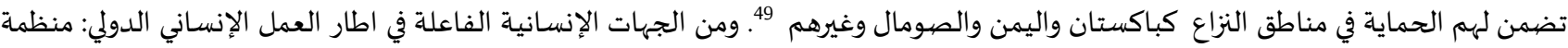

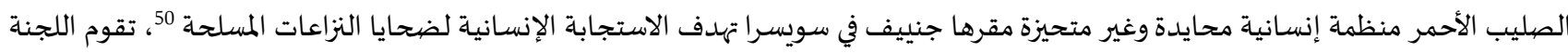
الدولية للصليب الأحمر بدور كبير في إطار تطبيق القانون الدولي الإنساني إذ تنص اتفاقياته على أن للجنائة الدولية للصبليب الأحمر زيارة الأسرى وتأمين

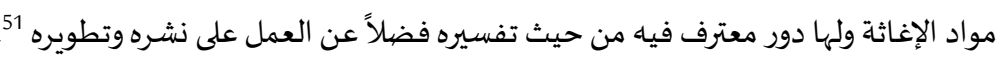
إن اللجنة الدولية للصليب الأحمر تعمل على مستوى دولي ووطني وتقوم على عدة ملهد مبادئ وهي: تتضمن المبادئ الإنسانية تقديم المساعدة للمتضررين في الأعمال القتالية لتخفيف الآمهم والحفاظ على الحياة والصحة وكرامة الإنسان لكافة الشعوب كما تلزم اتفاقيات جنيف الأربع

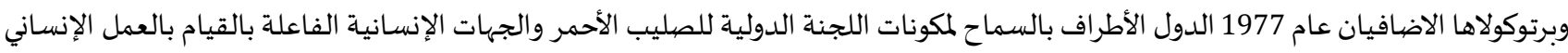

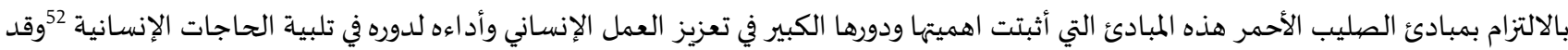

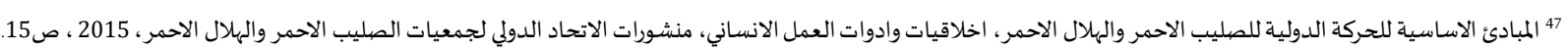

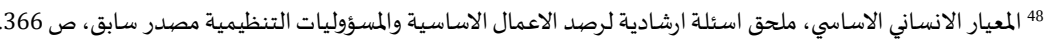
9 يوسف برقوق، مصدر سابق، ص 290. 50 بريز فتاح يونس، دور المنظمات الانسانية اثناء النزاعات الداخلية المسلحة (الصليب الاحمر نموذجا)، مجلة كلية القانون للعان

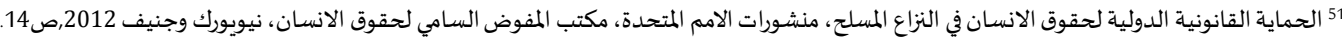

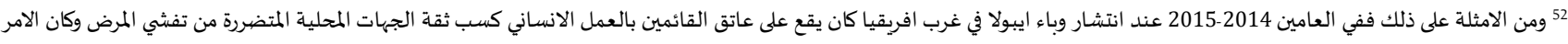

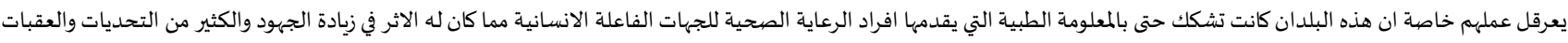

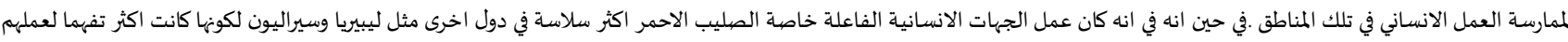

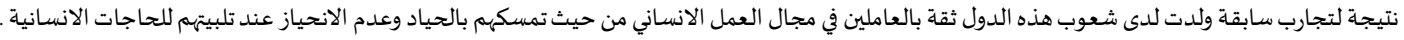


أكدت قرارات الأمم المتحدة أهمية هذه المبادئ لممارسة العمل الإنساني منها القرار رقم 182/46 في 1991 الذي يتضهمن تقديم الأمم المتحدة

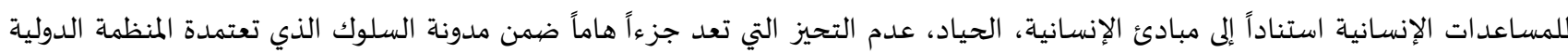
للصليب الأحمر عند ممارستها لمهمتها الإنسانية.

من الامثلة على تطبيق مبدء عدم التحيز وتقديم المساعدات الإنسانية بشكل موضيوعي بغض لمهنيالإنسانيه النظر عن العرق، الدين، الطائفة، العقيدة

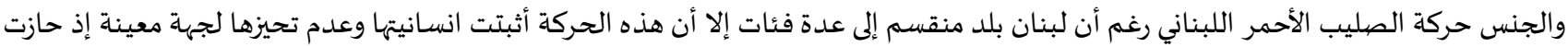

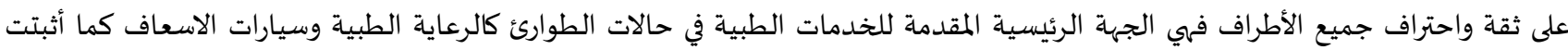

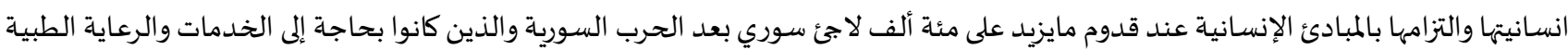

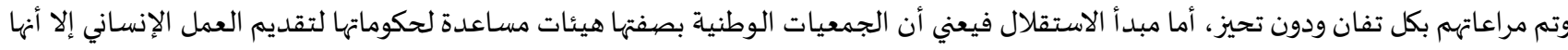

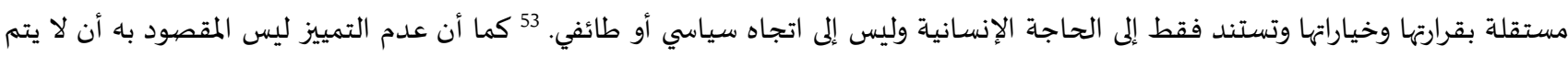

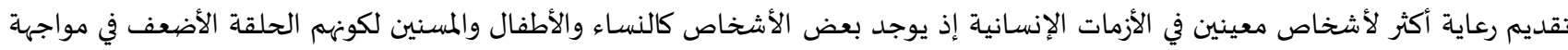

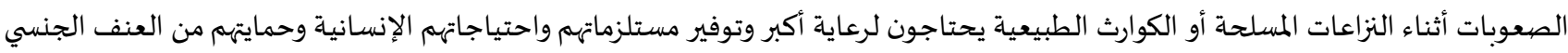

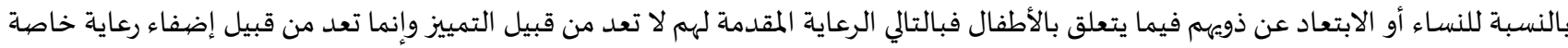

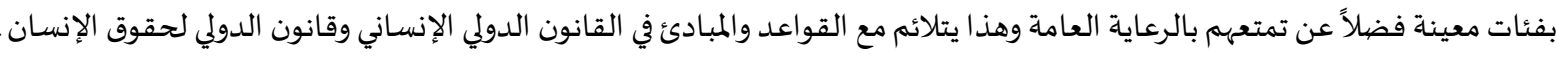

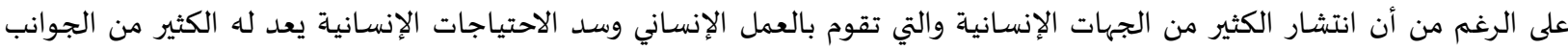

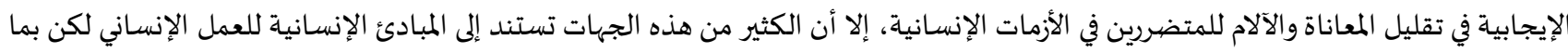

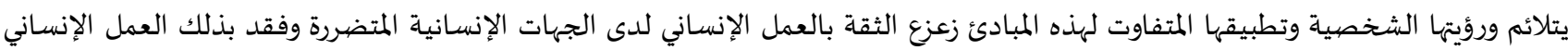

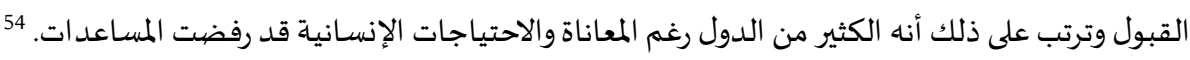
فأصبحت المبادئ الإنسانية لوحدها لا تكفي لكسب ثقدة الناس المتضررين فضيلاً عن أن مظاهر التسلح للعمليات الإنسانية قد كان سبباً في فقدان مقبولية الجهات الإنسانية الفاعلة.

المطلب الثالث: مدونة قواعد السلوك لمنظمة الصليب الأحمرفي حالات الطوارئ الصحية العامة

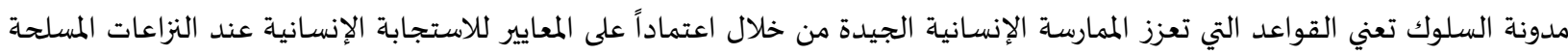

قواعد السلوك التي تسير بموجبها منظمة الصليب الأحمر عند قيامها بعملها الإنساني هي: أولوية الحاجات الإنسـانية: إن الحق في الحصيول على المساعدات الإنسانية يعد مبدأ إنساني أساسي. عدم التمييز عند تقديم المساعدات الإنسانياة.

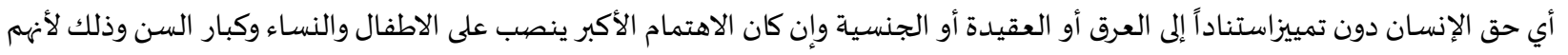

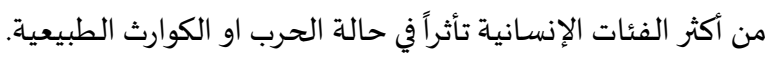
ستقلال الجهات الإنسانية الفاعلة عن دعم أي موقف ديني أوسياسي. استقلال الجهات الفاعلة عن الحكومات عند تقديم المساعدات الإنسانية إذ أن عمل هذه الجهات يقوم على الاستجابة للحاجات الإنسانيات الهانية وبشكل مستقل عن المصالح السياسية. 56 على الجهات الإنسانية الفاعلة احترام العادات والثقافات للبلد التي تقدم لها المساعدات الإنسانية. أن يتم العمل الإنساني الدولي بالمشاركة مع الجهات المحلية عند الاستجابة للحاجات الإنسانية. تفعيل طرق لاشراك المستفيدين من المساعدات الإنسانية في تصميم برامج المساعدة وإدارتها وإعادة تأهيلهم. يقوم العمل الإنساني على تلبية الاحتياجات الإنسانية الطارئة فضلاً عن العمل على برامج تساعد على تطوير وسائل عيش جديدة ومستدامة. 57.

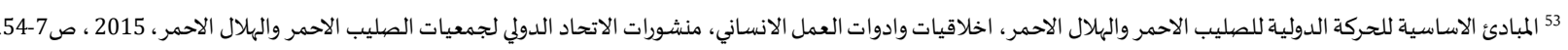

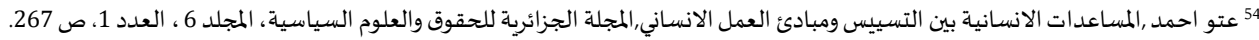

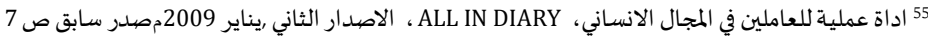

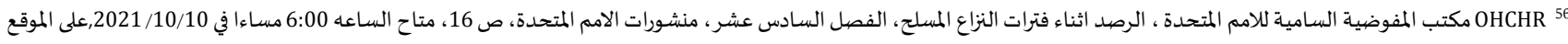

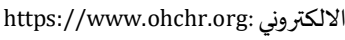
57 المعيار الانساني الاساسي، ملحق اسئلة ارشادية لرصد الاعمال الاساسية والمسؤوليات التنظيمية، مصدر السابق، ص 363 
إن الجهات الفاعلة الإنسانية في مجال العمل الإنساني الدولي تكون كحلقة وصل بين المانحين والمستفيدين بكل نزاهة ووضوح إذ تقدم تقارير

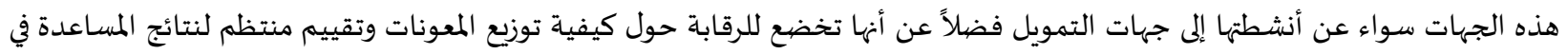
الأزمات الإنسانية أن يتم ممارسة العمل الإنساني وتقديم المساعدات الإنسـانية بطريقة تحفظ كرامة المتضررين عند استخدام الأنشطة الإعلامية والدعائية للتعبير عن هذه المساعدات فضلاً عن نقل صورتهم وحجم الأزمة الإنسانية التي يعانون منها بكل موضيوعية إلى وسائل الإعلام. 59

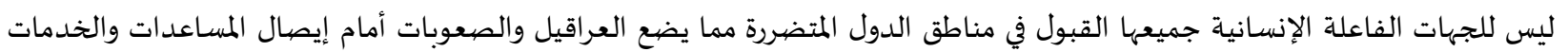

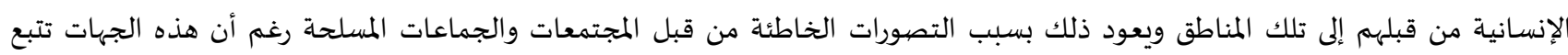

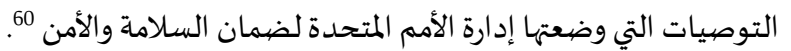

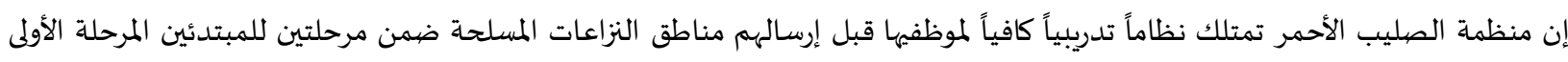

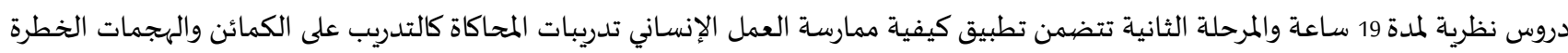

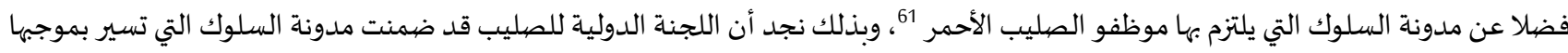

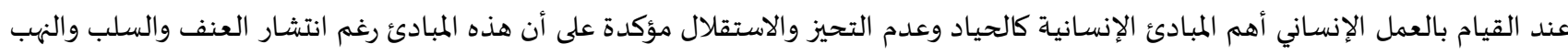

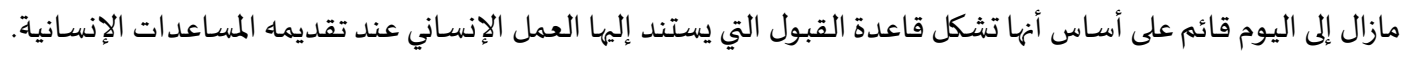

الخاتمة:

تناولنا من خلال بحثنا العمل الإنساني الدولي خلال أو عند انتشار الأوبئة وقد توصلنا إلى جملة من الاستنتاجات ثم لحقناها بجملة من الاقتراحات وذلك على النحو الاتي:

يقصيد بالعمل الإنساني التدابير التي يتم اتخاذها لتخفيف الآم ومراعاة السكان المتضررين من جراء النزاعات المسلحة أو الكوارث الطبيعة. تقع مسؤولية تلبية الاحتياجات الإنسانية للمتضررين على عاتق الدولة الموجودين فوق إقليمها وفي حالة عجزها عن ذلك لابد من تدخل الجهات الإنسانية الفاعلة لسد تلك الاحتياجات. تواجه اليوم العمل الإنساني والقائمين تحديات كبيرة جداً تعوق ممارستة وتعرضهم للاعتقال والتعذيب والخطف، من أهم هذه التحديات

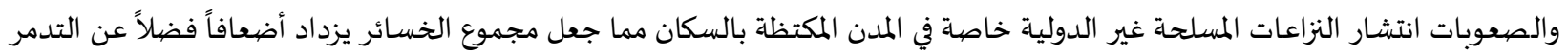

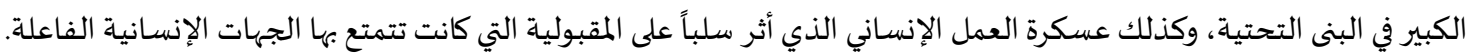
أن منظمة الصحة العالمية هي الوكالة الدولية المتخصصة في وضيع التوصيات اللازمة لمجابهة الأوبئة والجوائح وإدارة مخاطر الطوارئ والأزمات بإجراءات التاهب والترصد والاستجابة. هذا وتقاس حالات الطوارئ الصحية العامة بما تخلفه من وفيات وأمراض وإعاقات. أن النزاعات المسلحة تؤثر بصورة مباشرة على صحة الإنسان فضلاً عن مساسها بكل حقوق الإنسان الأخرى وتدميرها للبنى التحتية خاصة

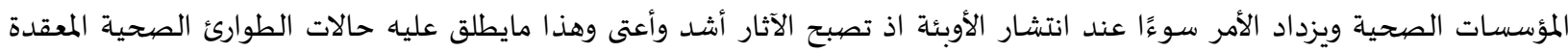
باجتماع النزاعات المسلحة وانتشار الأوبئة. تعد منظمة الصليب الأحمر من أكثر الجهات الفاعلة الإنسانية مقبولية لدى الجماعات المتنازعة لإثباتها المهنية الجيدة في العمل الإنساني

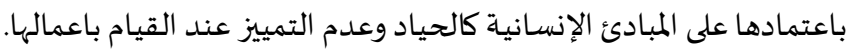
تعني مدونة السلوك للعمل الإنساني مجموعة من القواعد الإنسانية الجيدة من خلال اعتماد أعلى المعايير للاستجابة الإنسانية عند النزاعات المسلحة والكوارث. ثانياً: الاقتراحات: إن العمل الإنساني وممارسته تعد من الصعوبة في النزاعات المسلحة سواء أكانت دولية أم غير دولية فالجبهات المتنازعاة تنظر للجههة المقابلة على

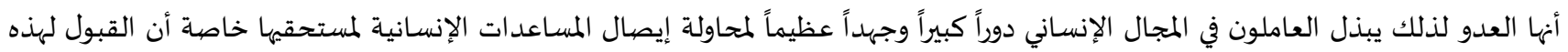

258 58

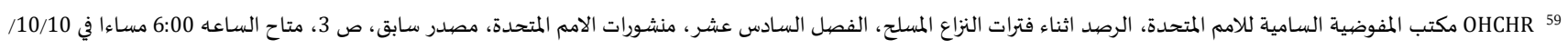

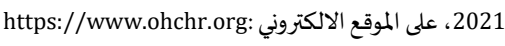

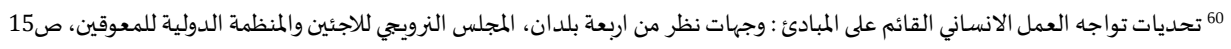

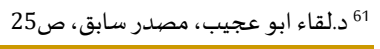


الجهات الفاعلة الإنسانية والمبني على فهم والإيمان بمبادئهم أصبح اليوم غير كاف لحماية القائمين بالعمل الإنساني مما تطلب توفير الحماية الفعلية

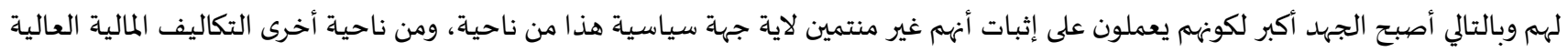

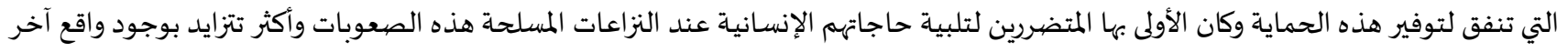

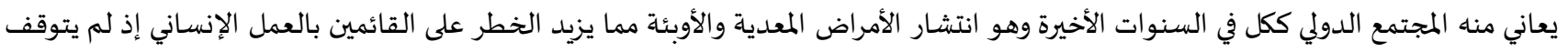

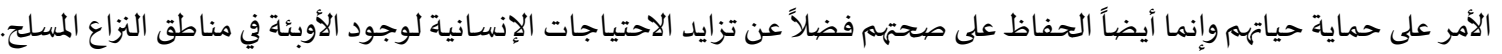
لذا نقترح ضمن هذا السياق مايلي:

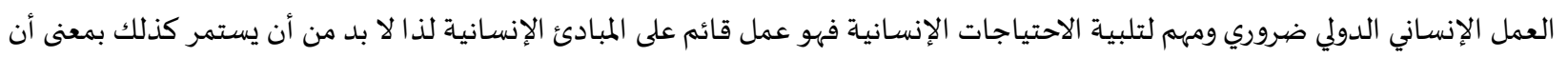
يبقى العاملون في المجال الإنساني بمظهر مدني وأن تسلم المساعدات والخدمات الإنسانية من قبلهم حصراً لا من الجهات الأمنية التي يستعينون . ب. لتقليل الخسائر والمخاطر يجب الاستعانة بالسكان المحليين انفسهم في إيصال المساعدات الإنسانية لمستحقيها ولكن بعد أن يتم تدريهم نظرياً وميدانياً حفاظاً على سلامتهم وسلامة القائمين بالعمل الإنساني.

عقد مؤتمرات دولية مخصصية لبحث معوقات العمل الإنساني في مناطق النزاع المسلح خاصية عند انتشار الأوبئة. تكثيف الجهود الإنسانية في الحوار والتفاوض مع الجهات المسلحة ومحاولة اعادة كسب الثقة بسلامة العمل الإنساني المحايد.

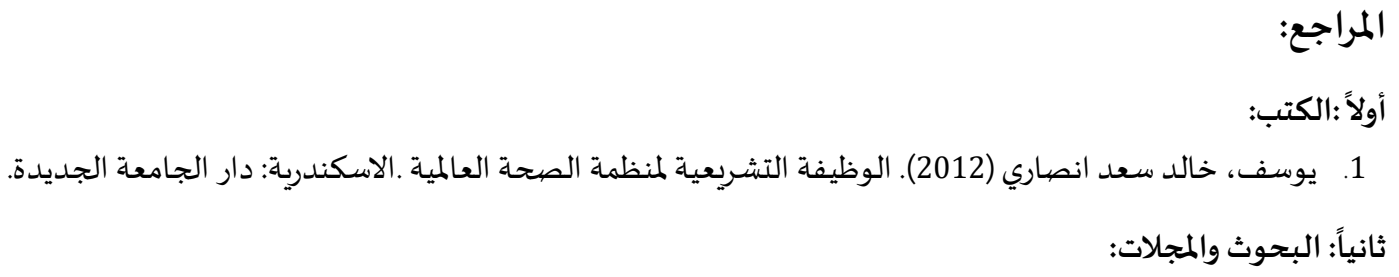

1. أحمد، عتو (2021). المسـاعدات الإنسانية بين التسييس ومبادئ العمل الإنساني. المجلة الجزائرية للحقوق والعلوم السياسية، 6(1)

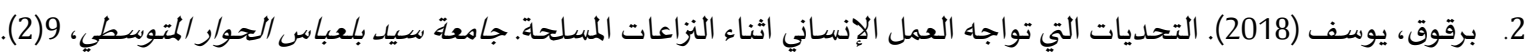
3. بيران، بيار (2001). الطوارئ الصحية في التحجمعات السكانية الكبيرة. منشورات اللجنة الدولية للصبليب الأحمر.

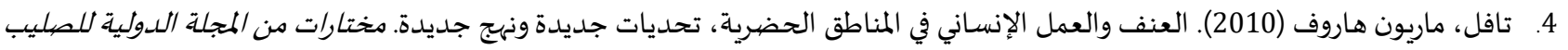

الأحمر،

5. حميد، هالة خالد (2021). إشكالية الأمن المائي في العلاقات التركية العراقية، دراسـة في اليات التعامل الامثل في قضية المياه. مجلة القضايا

السياسية، (65).

6 س. سالم، ماجد صدام (2019). أثر الارهاب على التنمية والأمن الغذائي في العراق. (عدد خاص للمؤتمر العلمي الدولي الثالث، كلية التربية الاساسية

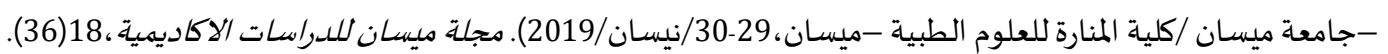

7.

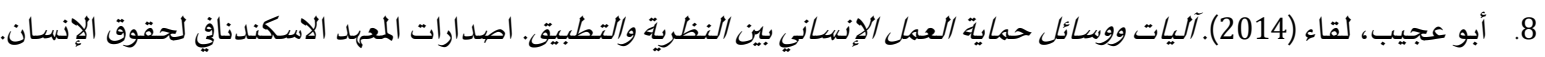

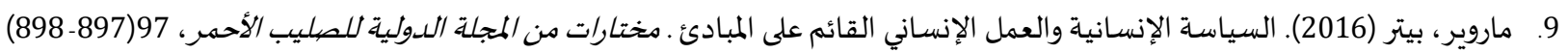

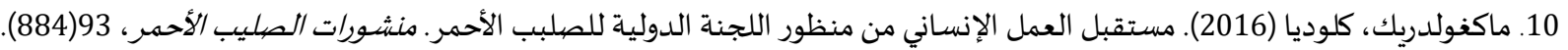
11. يونس، بريز فتاح (2016). دور المنظمات الإنسانية اثناء النزاعات الداخلية المسلحة (الصليب الأحمر نموذجا). مجلة كلية القانية القانون للعلوم

القانونية والسياسية، (16) بونئ)

ثالثاً: منشورات موقع الأمم المتحدة:

1. المعيار الإنساني الاساسي، ملحق اسئلة ارشادية لرصد الأعمال الاساسية والمسؤوليات التنظيمية، متاح على الموقع الالكتروني تمت الزيارة

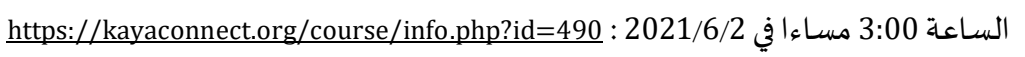

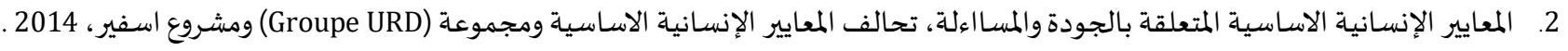

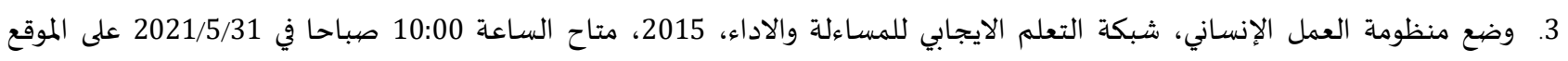

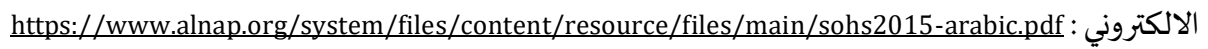
4. الجنـة الدائمة المشتركة بين الوكالات ومكتب تنسيق الشؤون الإنسانية التابع للامم المتحدة 2007، وقت الزيارة 9:00، في /2021/8، 2015، متاح على http:ochaonline.un.orglcmcs/guideline: الموقع الالكترونين 
5. المشاورة الاقليمية للشرق الاوسط وشمال افريقيا، القمة العالمية للعمل الإنساني، عمان -الاردن، 202115/5/2015. تمت الزيارة في 4:00 مسـاءا في

https://www.alnap.org/system/files/content/resource/files/main/scoping-paper-mena-regional- 2021/6/11 على الموقع الالكتروني: consultation-\%5Barabic\%5D.pdf

6. OHCHR مكتب المفوضية السـامية للامم المتحدة، الرصد اثناء فترات النزاع المسلح، الفصل السادس عشر، منشورات الأمم المتحدة، الساعة https://www.ohchr.org:6:00 مساءا في 2021/10/10، متاح على الموقع الالكتروني

رابعاً: منشورات المنظمة الدولية للصليب الأحمر:

1. برنامج القانون الدولي لمواجهة الطوارئ في الاتحاد الدولي لجمعيات الصليب الأحمر والهلال الأحمر، منشورات الاتحاد الدولي لجمعيات الصليب

الأحمر والهلال الأحمر، نشرة رقم 11 الخاصة بالقوانين والقوائه والقواعد والمبادئ الدولية الخاصة بالاستجابة للكوارث، 2008.

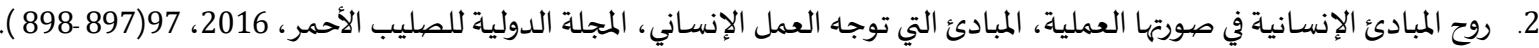

3. شرح مسودة الارشادات المتعلقة بتسهيل وتنظيم المساعدات الدولية للاغاثة والانتعاش الدولي على الصعيد المحلي في حالات الكوارث، الاتحاد

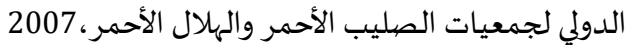

4. المبادئ الاساسية للحركة الدولية للصليب الأحمر والهلال الأحمر، اخلاقيات وادوات العمل الإنساني، منشورات الاتحاد الدولي لجمعيات

الصليب الأحمر والهلال الأحمر، 2015.

خامساً: الوثائق الدولية:

1. بيان الاجتماع الرابع للجنة الطوارئ المعنية باللوائح الصحية العالمية 2005 بشان فاشية المرض الناجم عن فيروس كورونا (COVID 19)، منظمة

الصحة العالمية، متاح في الساعة السادسة في 2021/10/8 على الموقع الإلكتروني:

https://www.who.int/ar/news/item/11-12-1441-statement-on-the-fourth-meeting-of-the-international-health-regulations-(2005)-emergency-committeeregarding-the-outbreak-of-coronavirus-disease-(covid-19)

$$
\begin{aligned}
& \text { 2. قرار الجمعية العامة للامم المتحدة رقم 51/37 في } 1982
\end{aligned}
$$

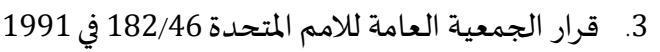

$$
\begin{aligned}
& \text { 4. قرار الجمعية العامة للامم المتحدة } 1325 \text { في } 2000
\end{aligned}
$$

5. معايير وممارسات الأمن الخاصة بموظفي المساعدات الإنسانية تدريب والدعوة لافساح المجال للعمل الإنساني، تقرير حول امن موظفي

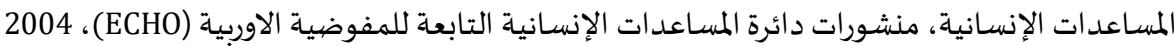

خامساً: المراجع الأجنبية:

1. Lucia Mullen Christina Potter, Llawrence O Gostin,Anita Cicero,Jennifer B Nuzzo (2020). An analysis of International Health Regulations Emergency committees and Publice Health Emergency of International Concern Designation, 5: e002502. 10.1136/bmjgh-2020-002502

2. Obijiofor Aginam (2002). International law and communicable diseases, Global Public Health and International Law. Bulletin of the World Health Organization, 80.

3. Legal Responses to Health Emergencies, The Law Library of congress, Global Legal Research Center (202)7076462(phone). (866)550-0442(fax). law@loc.gov http://www.law.gov.

4. Muhammad A.Soghaier ,Khawaja MI Saeed, \& Khushhal K.Zaman (2015). Public Health Public Health Emergency of International Concern (PHEIC)twice in 2014; Polio and Ebola at top, AIMS Public Health, 2(2): 218-222. https://doi.org/10.3934/publichealth.2015.2.218

سـادساً: المو اقع الإلكترونية:

1. Advancing the right to health : the vital role of law ,Public health emergencies SUMMARY POINTS ,p 169

https://www.researchgate.net/publication/324531402_Advancing_the_Right_to_Health-The_Vital_Role_of_Law 


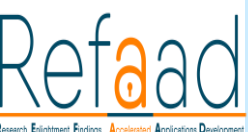

www.refaad.com
المجلة الدولية للدراسات القانونية والفقهية المقارنة

International Journal of Legal and Comparative Jurisprudence Studies (LCJS)

Journal Homepage: https://www.refaad.com/views/LCJS/Home.aspx ISSN: 2708-6607(Online) 2708-6593(Print)

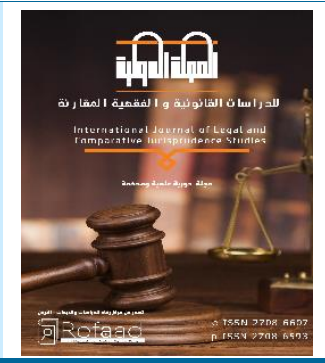

\title{
International action in public health emergencies (The role of the International Committee of the Red Cross as a model)
}

\author{
Wahag Khder Abbas \\ Assistant Professor, College of Law, University of Basra, Iraq \\ wahag.khder@gmail.com
}

Received: 6/9/2021 Revised: 30/9/2021 Accepted: 20/10/2021 DOI: https://doi.org/10.31559/LCJS2021.2.3.3

Abstract: Humanitarian work includes a set of measures intended to alleviate the pain and suffering of those affected by humanitarian crises, whether armed conflicts or natural disasters. Those undertaking humanitarian work rely on humanitarian principles such as neutrality, impartiality and independence to obtain acceptance from the conflicting parties to ensure the timely delivery of humanitarian aid and services. Deserving it, today however, humanitarian work faces many challenges and obstacles, such as kidnappings and violence against people doing humanitarian work, as well as the spread of epidemics and pandemics in unsafe environments, which endangers the lives of those doing humanitarian work and many difficulties that prevented the timely delivery of the humanitarian needs of those affected.

Keywords: Humanitarian work; public health emergencies; humanitarian response standards; code of conduct.

\section{References:}

1. Abw 'jyb, Lqa' (2014). Alyat Wwsa'l Hmayh Al'ml Alensany Byn Alnzryh Walttbyq. Asdarat Alm'hd Alaskndnafy Lhqwq Alensan.

2. Al'thmany, Nshwan (2020). Nsa' Alymn Wa'ba' Mda'fh Fy Zmn Kwrwna. Mnshwrat Alljnh Aldwlyh Llslyb Alahmralensany, Al'dd 67.

3. Ahmd, 'tw (2021). Almsa'dat Alensanyh Byn Altsyys Wmbad' Al'ml Alensany. Almjlh Aljza'ryh Llhqwq Wal'lwm Alsyasyh, 6(1).

4. Brqwq, Ywsf (2018). Althdyat Alty Twajh Al'ml Alensany Athna' Alnza'at Almslhh. Jam't Syd Bl'bas Alhwar Almtwsty, $9(2)$.

5. Byran, Byar (2001). Altwar' Alshyh Fy Altjm'eat Alskanyh Alkbyrh. Mnshwrat Alljnh Aldwlyh Llslyb Alahmr.

6. Hmyd, Halh Khald (2021). Eshkalyh Alamn Alma'y Fy Al'laqat Altrkyh Al'raqyh, Drash Fy Alyat Alt'aml Alamthl Fy Qdyh Almyah. Mjlt Alqdaya Alsyasyh, (65).

7. Makghwldryk, Klwdya (2016). Mstqbl Al'ml Alensany Mn Mnzwr Alljnh Aldwlyh Llslbb Alahmr. Mnshwrat Alslyb Alahmr, 93(884).

8. Marwyr, Bytr (2016). Alsyash Alensanyh Wal'ml Alensany Alqa'm 'la Almbad'. Mkhtarat Mn Almjlh Aldwlyh Llslyb Alahmr, 97(897- 898).

9. Salm, Majd Sdam (2019). Athr Alarhab 'la Altnmyh Walamn Alghda'y Fy Al'raq. ('dd Khas Llm'tmr Al'Imy Aldwly Althalth, Klyt Altrbyh Alasasyh -Jam't Mysan /Klyt Almnarh Ll'lwm Altbyh -Mysan,29-30/Nysan/2019). Mjlt Mysan Lldrasat Alakadymyh,18(36).

10. Tafl, Marywn Harwf (2010). Al'nf Wal'ml Alensany Fy Almnatq Alhdryh, Thdyat Jdydh Wnhj Jdydh. Mkhtarat Mn Almjlh Aldwlyh Llslyb Alahmr, 92(878).

11. Ywns, Bryz Ftah (2016). Dwr Almnzmat Alensanyh Athna' Alnza'at Aldakhlyh Almslhh (Alslyb Alahmr Nmwdja). Mjlt Klyt Alqanwn Ll'lwm Alqanwnyh Walsyasyh, (16). 